\title{
Index to Volume 33, 2017
}

\author{
Contents of Volume 33, Numbers 1-4
}

Articles, see Author Index

Book Reviews 1087, 1091,

Editorial Collaborators 1093

Index 1101

Editorial/Preface 301, 571

\begin{abstract}
Author Index
Abreu D.A., See Sang. H.

Arroway, P., See Sang, H.

Ashmead, R., Slud, E., Hughes, T. Adaptive Intervention Methodology for Reduction of

Respondent Contact Burden in the American Community Survey . . . . . . . . . . . . . . . . . . . . . 901-919

Barros da Silva, E. A., See Cohen Martelotte, M.

Barthélemy, J., See Suesse, T.

Bates, N. Morris Hansen Lecture: Hard-to-Survey Populations and the U.S. Census: Making

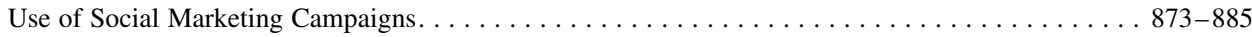

Bell, P., See Chipperfield, J.

Bell, R. Unit Root Properties of Seasonal Adjustment and Related Filters: Special Cases . . . . . . . . . 1-14

Berzofsky, M.E., See Edwards, S.

Beullens, K., See Loosveldt, G.

Beyler, A., See Beyler N.

Beyler, N., Beyler, A. Adjusting for Measurement Error and Nonresponse in Physical Activity

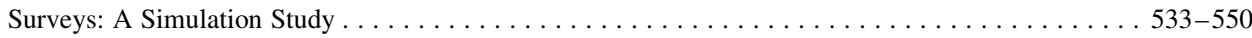

Bianchi, A., Biffignandi, S., Lynn, P. Web-Face-to-Face Mixed-Mode Design in a Longitudinal

Survey: Effects on Participation Rates, Sample Composition, and Costs . . . . . . . . . . . . . . . 385-408

Biemer, P.P., See Edwards, S.

Biffignandi, S., See Bianchi, A.

Blohm, M., See Gweon, H.

Boeschoten, L. Oberski, D., de Waal, T. Estimating Classification Errors under Edit Restrictions

in Composite Survey-Register Data Using Multiple Imputation Latent Class Modelling (MILC) . . . 921-962

Boeschoten, L., Vink, G., Hox, J.J.C.M. How to Obtain Valid Inference under Unit Nonresponse? . . 963-978

Brick, J.M., Tourangeau, R. Responsive Survey Designs for Reducing Nonresponse Bias . . . . . . . $735-752$

Brown, J., See Chipperfield, J.

Beullens, K., See Vandenplas, C.

Buellens, B., Van den Brakel, J.A. Comparing Two Inferential Approaches to Handling

Measurement Error in Mixed-Mode Surveys. . . . . . . . . . . . . . . . . . . . . . 513-531

Burger, J., Perryck, K., Schouten, B. Robustness of Adaptive Survey Designs to Inaccuracy

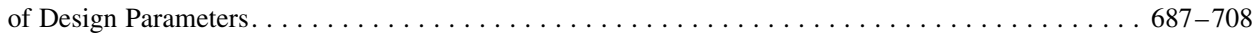

Calviño, A. A Simple Method for Limiting Disclosure in Continuous Microdata Based on

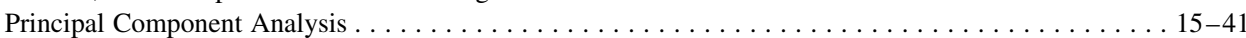

Castro Souza, R., See Cohen Martelotte, M.

Chipperfield, J., Brown, J., Bell, P. Estimating the Count Error in the Australian Census . . . . . . . . . 43-59

Chowdhury, S.R., See Mirel, L.B.

Chun, A.Y., Schouten, B., Wagner, J. JOS Special Issue on Responsive and Adaptive
\end{abstract}

Survey Design: Looking Back to See Forward - Editorial. . . . . . . . . . . . . . . . . . . . . . . 571-577 
Cohen Martelotte, M., Castro Souza, R., Barros da Silva, E. A. Design of Seasonal Adjustment

Filter Robust to Variations in the Seasonal Behaviour of Time Series . . . . . . . . . . . . . $155-186$

D’Aló, M., Falorsi, S., Solari, F. Space-Time Unit-Level EBLUP for Large Data Sets . . . . . . . . . . 61-77

De Leeuw, E., See Eckman, S.

De Waal, T., See Boeschoten, L.

Durrant, G.B., Maslovskaya, O., Smith, P.W.F. Using Prior Wave Information and Paradata:

Can They Help to Predict Response Outcomes and Call Sequence Length in a Longitudinal Study? . 801-833

Early, K. Mankoff, J., Fienberg, S.E. Dynamic Question Ordering in Online Surveys . . . . . . . . . 6 625-657

Eckman, S., de Leeuw, E. Editorial - Special Issue on Total Survey Error (TSE) . . . . . . . . . . . . 301

Edwards, B. Discussion . . . . . . . . . . . . . . . . . . . . . . . . . . . . . . . . . 887-890

Edwards, S., Berzofsky, M.E., Biemer, P.P. Effect of Missing Data on Classification

Error in Panel Surveys. . . . . . . . . . . . . . . . . . . . . . . . . . . . . . . . . . . . . 551-570

Falorsi, S., See D’Aló, M.

Fienberg, S., See Early, K.

Gal, I., Ograjenšek, I. Official Statistics and Statistics Education: Bridging the Gap . . . . . . . . . . 79-100

Gweon H., Schonlau, M., Kaczmirek, L., Blohm, M., Steiner, S. Three Methods for Occupation

Coding Based on Statistical Learning . . . . . . . . . . . . . . . . . . . . . . . . . . . 101-122

Holmberg, A., See Reid, G.

Hox, J.J.C.M., See Boeschoten, L.

Huang, N., Wimalaratne, W., Pollard, B. The Effects of the Frequency and Implementation

Lag of Basket Updates on the Canadian CPI. . . . . . . . . . . . . . . . . . . . . . . . . . . . . . . . 979-1004

Hughes, T., See Ashmead, R.

Jacobsen, A. L. Discussion. . . . . . . . . . . . . . . . . . . . . . . . . . . . . . . . . . . . . 891-899

James, G.G., See Smith, P.A.

Kaczmirek, L., See Gweon, H.

Kaminska, O., Lynn, P. The Implications of Alternative Allocation Criteria in Adaptive

Design for Panel Surveys. . . . . . . . . . . . . . . . . . . . . . . . . . . . . . . . . . . . . . 781-799

Kaminska, O., Lynn, P. Survey-Based Cross-Country Comparisons Where Countries

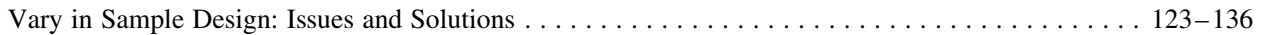

Kaputa, S.J., See Thompson, K.J.

Keller, A.D., See Mulry, M.

Kenyon, S., See Spencer, B.D.

Keusch, F., See Liu, M.

Lamas, A.C., See Sang, H.

Lee, S., Suzer-Gurtekin, T., Wagner, J., Valliant, R. Total Survey Error and Respondent

Driven Sampling: Focus on Nonresponse and Measurement Errors in the Recruitment

Process and the Network Size Reports and Implications for Inferences. . . . . . . . . . . . . . 335-366

Lewis, T. Univariate Tests for Phase Capacity: Tools for Identifying When to Modify a

Survey's Data Collection Protocol . . . . . . . . . . . . . . . . . . . . . . . . . . . . . . . 601-624

Liu, M., Keusch, F. Effects of Scale Direction on Response Style of Ordinal Rating Scales . . . . . 137-154

Loong, B., Rubin, D.B. Multiply-Imputed Synthetic Data: Advice to the Imputer. . . . . . . . . . . 1005-1019

Loosveldt, G., See Vandenplas, C.

Loosveldt, G., Beullens, K. Interviewer Effects on Non-Differentiation and Straightlining

in the European Social Survey

Lopéz, A.J., See Vicente, M.R.

Lopiano, K.K., See Sang, H.

Lundquist, P., See Särndal, C.-E.

Lynn, P., See Bianchi, A.

Lynn, P., See Kaminska, O.

Maslovskaya, O., See Durrant, G.B.

May, J., See Spencer, B.D.

Mankoff, J., See Early, K.

McCarthy, J., Wagner, J., Sanders, H.L. The Impact of Targeted Data Collection on

Nonresponse Bias in an Establishment Survey: A Simulation Study of Adaptive Survey Design . . . 857-871

Mirel, L.B., Chowdhury, S.R. Using Linked Survey Paradata to Improve Sampling

Strategies in the Medical Expenditure Panel Survey . . . . . . . . . . . . . . . . . . . . . . . 367-383

Mokhtarian, P., See Suesse, T. 
Mulry, M. Keller, A.D. Comparison of 2010 Census Nonresponse Follow-Up Proxy

Responses with Administrative Records Using Census Coverage Measurement Results. . .

Namazi-Rad, M.-R., See Suesse, T.

Oberski, D., See Boeschoten, L.

Ograjenšek, I., See Gal, I.

Paiva, T., Reiter, J.P. Stop or Continue Data Collection: A Nonignorable Missing Data Approach for Continuous Variables . . . . . . . . . . . . . . . . . . . . . . . . . . . . . . . . . . . . . . . . . . . . . 579-599

Pollard, B., See Huang, N.

Perryck, K., See Burger, J.

Plewis, I., Shlomo, N. Using Response Propensity Models to Improve the Quality of Response

Data in Longitudinal Studies . . . . . . . . . . . . . . . . . . . . . . . . . . . . . 753-779

Reid, G., Zabala, F., Holmberg, A. Extending TSE to Administrative Data: A Quality

Framework and Case Studies from Stats NZ . . . . . . . . . . . . . . . . . . . . . . . 477-511

Reiter, J.P., See Paiva. T.

Roberts, C., Vandenplas, C. Estimating Components of Mean Squared Error to Evaluate

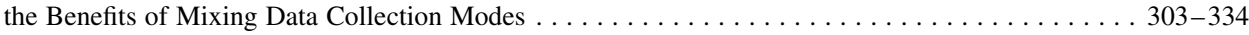

Rothbaum, J. Bridging a Survey Redesign Using Multiple Imputation: An Application

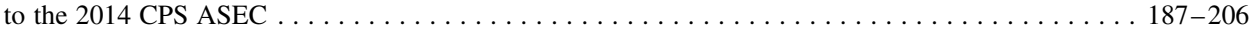

Rubin, D.B., See Loong, B.

Sanders, H.L., See McCarthy, J.

Sang, H., Lopiano, K.K., Abreu D.A., Lamas, A.C., Arroway, P., Young, L.J. Adjusting for

Misclassification: A Three-Phase Sampling Approach . . . . . . . . . . . . . . . . . . . . . . . . 207-222

Schmidt, T., Sieber, S. The Influence of an Up-Front Experiment on Respondents' Recording

Behaviour in Payment Diaries: Evidence from Germany . . . . . . . . . . . . . . . . . . . . . 4 427-454

Schonlau, M., See Gweon, H.

Schouten, B., See Chun, A.Y.

Schouten, B., See Burger, J.

Seeskin, Z., See Spencer, B.D.

Shlomo, N., See Plewis, I.

Sieber, S., See Schmidt, T.

Slud, E., See Ashmead, R.

Smith, P.A., James, G.G. Changing Industrial Classification to SIC (2007) at the UK Office

for National Statistics . . . . . . . . . . . . . . . . . . . . . . . . . . . . . . . . . . . . . . . . . . . 223-247

Smith, P.W.F., See Durrant, G.B.

Solari, F., See D’Aló, M.

Spencer, B.D., May, J., Kenyon, S., Seeskin, Z. Cost-Benefit Analysis for a Quinquennial Census:

The 2016 Population Census of South Africa . . . . . . . . . . . . . . . . . . . . . . . . . . . . 249-274

Steiner, S., See Gweon, H.

Suesse, T., Namazi-Rad, M.-R., Mokhtarian, P., Barthélemy, J. Estimating Cross-Classified

Population Counts of Multidimensional Tables: An Application to Regional Australia to Obtain

Pseudo-Census Counts. . . . . . . . . . . . . . . . . . . . . . . . . . . . . . . . . . . . . . . . . 1021-1050

Suzer-Gurtekin, T., See Lee, S.

Särndal, C.-.E., Lundquist, P. Inconsistent Regression and Nonresponse Bias: Exploring

Their Relationship as a Function of Response Imbalance. . . . . . . . . . . . . . . . . . . . . . 709-734

Théberge, A. Estimation when the Covariance Structure of the Variable of Interest is

Positive Definite . . . . . . . . . . . . . . . . . . . . . . . . . . . . . . . . . . . . . . . . . 275-299

Thompson, K.J., Kaputa, S.J. Investigating Adaptive Nonresponse Follow-up Strategies

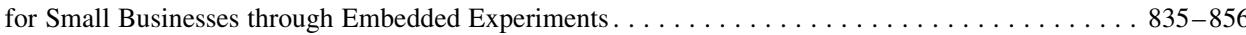

Tourangeau, R., See Brick, M.J.

Valliant, R., See Lee, S.

Van den Brakel, J.A., See Buelens, B.

Vandenplas, C., See Roberts, C.

Vandenplas, C., Loosveldt, G, Beullens, K. Fieldwork Monitoring for the European Social Survey:

An illustration with Belgium and the Czech Republic in Round $7 \ldots \ldots \ldots \ldots \ldots \ldots$. . . . . . 659-686

Vicente, M.R., Lopéz, A.J. Figuring Figures: Exploring Europeans’ Knowledge of

Official Economic Statistics . . . . . . . . . . . . . . . . . . . . . . . . . . . . . . . . 1051-1085

Vink, G., See Boeschoten, L. 
Wagner, J., See Chun, A.Y.

Wagner, J. See Lee, S.

Wagner, J., See McCarthy, J.

Wimalaratne, W., See Huang, N.

Young, L.J., See Sang, H.

Zabala, F., See Reid, G.

\section{Book Reviews}

Modern Data Science with R.

Nathan Cruze . . . . . . . . . . . . . . . . . . . . . . . . . . . . . . . . . . . . . . . 1087-1089

Statistical Analysis of Questionnaires: A Unified Approach Based on R and Stata.

Morgan Earp. . . . . . . . . . . . . . . . . . . . . . . . . . . . . . . . . . 1091-1092

Printed in December 2017 